\title{
Linear polarization of submillimetre masers
}

\section{Tracing magnetic fields with ALMA}

\author{
A. F. Pérez-Sánchez ${ }^{1}$ and W. H. T. Vlemmings ${ }^{2}$ \\ 1 Argelander Institute for Astronomy, University of Bonn, Auf dem Hügel 71, 53121 Bonn, Germany \\ e-mail: aperez@astro.uni-bonn.de \\ 2 Chalmers University of Technology, Onsala Space Observatory, 43992 Onsala, Sweden \\ e-mail: wouter .vlemmings@chalmers.se
}

Received 14 November 2012 / Accepted 4 January 2013

\section{ABSTRACT}

\begin{abstract}
Context. Once ALMA full polarization capabilities are offered, it will become possible to perform detailed studies of polarized maser emission towards star-forming regions and late-type stars, such as (post-) asymptotic giant branch stars and young planetary nebulae. To derive the magnetic field orientation from maser linear polarization, a number of conditions involving the rate of stimulated emission $R$, the decay rate of the molecular state $\Gamma$, and the Zeeman frequency $g \Omega$ need to be satisfied.

Aims. The goal of this work is to investigate if $\mathrm{SiO}, \mathrm{H}_{2} \mathrm{O}$ and $\mathrm{HCN}$ maser emission within the ALMA frequency range can be detected with observable levels of fractional linear polarization in the regime where the Zeeman frequency is greater than the stimulated emission rate.

Methods. We used a radiative transfer code to calculate the fractional linear polarization as a function of the emerging brightness temperature for a number of rotational transition of $\mathrm{SiO}, \mathrm{H}_{2} \mathrm{O}$ and $\mathrm{HCN}$ that have been observed to display maser emission at submillimetre wavelengths. We assumed typical magnetic field strengths measured towards galactic star-forming regions and circumstellar envelopes of late-type stars from previous VLBI observations. Since the Landé g-factors have not been reported for the different rotational transitions we modelled, we performed our calculations assuming conservative values of the Zeeman frequency for the different molecular species.

Results. Setting a lower limit for the Zeeman frequency that still satisfies the criteria $g \Omega>R$ and $g \Omega>\Gamma$, we find fractional polarization levels of up to $13 \%, 14 \%$ and $19 \%$ for the higher $J$ transitions analysed for $\mathrm{SiO}, \mathrm{H}_{2} \mathrm{O}$ and $\mathrm{HCN}$, respectively, without considering anisotropic pumping or any other non-Zeeman effect. These upper limits were calculated assuming a magnetic field oriented perpendicular to the direction of propagation of the maser radiation.

Conclusions. According to our results, $\mathrm{SiO}, \mathrm{H}_{2} \mathrm{O}$, and $\mathrm{HCN}$ maser emission within the ALMA frequency range can be detected with suitable linear polarization to trace the magnetic field structure towards star-forming regions and late-type stars even if the detected polarization has been enhanced by non-Zeeman effects.
\end{abstract}

Key words. stars: AGB and post-AGB - masers - stars: magnetic field - polarization - stars: formation - submillimeter: stars

\section{Introduction}

Polarized maser emission has been detected towards starforming regions (SFRs) and expanding circumstellar envelopes (CSE) of late-type stars such as (post-) asymptotic giant branch (AGB) stars and young planetary nebulae (PNe; e.g. Alves et al. 2012; Amiri et al. 2012; Leal-Ferreira et al. 2012; Vlemmings et al. 2011, 2006b). Both single-dish and interferometric observations have revealed that silicon monoxide $(\mathrm{SiO})$, water $\left(\mathrm{H}_{2} \mathrm{O}\right)$, hydrogen cyanide $(\mathrm{HCN})$, hydroxyl $(\mathrm{OH})$, and methanol $\left(\mathrm{CH}_{3} \mathrm{OH}\right)$, among others, can naturally generate polarized maser emission in these enviroments (e.g. Vlemmings et al. 2006b; Surcis et al. 2011; Fish \& Reid 2007b; Herpin et al. 2006; Kemball et al. 2009). In particular, interferometric observations of the masers at radio wavelengths have become a useful tool for studying the magnetic field in and around SFRs and late-type stars (e.g. Fish \& Reid 2007a; Amiri et al. 2010). At shorter wavelengths, new instruments will enable the study of maser radiation from higher vibrationally-excited rotational transitions. In particular, the Atacama Large Millimeter/submillimeter Array (ALMA) has recently started the first scientific observations. Soon all of its capabilities, including polarimetry, will be available, providing more than an order of magnitude improvement in sensitivity and resolution. In the ALMA frequency range, a number of $\mathrm{SiO}, \mathrm{H}_{2} \mathrm{O}$ and $\mathrm{HCN}$ maser transitions that belong to vibrationally-excited levels up to $v=3$ can be detected. The population inversion of rotational states belonging to different molecular species, or within different vibrational levels of a particular species, depends on the physical conditions of the emitting regions. By observing the maser polarization at different frequencies towards an extended source, we can hence constrain the magnetic field properties, i.e. field strength and/or direction, throughout large regions within the observed source.

According to the Zeeman interpretation, the maser polarization strongly depends on the ratio between the Zeeman frequency $(g \Omega)$, the rate of stimulated emission $(R)$, and the rate of the decay of the molecular state $(\Gamma)$. The Zeeman splitting induced by a magnetic field depends on the molecule's shell structure. For example, since $\mathrm{SiO}, \mathrm{H}_{2} \mathrm{O}$ and $\mathrm{HCN}$ are nonparamagnetic, closed-shell molecules, $g \Omega$ is expected to be less than the intrinsic line width $\Delta \omega$. Maser polarization theory (e.g. Western \& Watson 1984) predicts fractional linear polarization $\left(p_{L}\right)$ levels of up to $100 \%$ for $J=1-0$ rotational transitions of diatomic molecules. These polarization levels can be reached 
in the presence of a magnetic field of a few Gauss in, for example, the $\mathrm{SiO}$ masing region in CSEs of late-type stars. But in the case of higher rotational transitions (i.e. $J=2-1, J=3-2$, etc.) theory predicts that unless anisotropic pumping is involved, the fractional linear polarization should decrease as the angular momentum number of the involved state increases.

Before the observed maser polarization can be related to an intrinsic magnetic field, it is necessary to evaluate the ratios between the maser parameters $R, g \Omega$ and $\Gamma$ for each single rotational transition detected with angular momentum higher than $J=1$. Here, we determine if the polarized maser radiation produced by molecular transitions of $\mathrm{SiO}, \mathrm{H}_{2} \mathrm{O}$ and $\mathrm{HCN}$ in the ALMA frequency range could generate detectable levels of fractional linear polarization, while still satisfying the criteria $(g \Omega>R$ and $g \Omega>\Gamma)$ for which the polarization direction is still directly related to the magnetic field. These maser transitions can then be used to infer the magnetic field structure towards SFRs and in the CSEs of (post-) AGB stars. To do this, we have run numerical models adapted from Nedoluha \& Watson (1992) to calculate the fractional linear polarization level that can be generated by the interaction of the molecular states of $\mathrm{SiO}, \mathrm{H}_{2} \mathrm{O}$ and $\mathrm{HCN}$ in the ALMA frequency range, with a magnetic field in the masing region.

In Sect. 2 we present a brief review of the maser polarization theory and in Sect. 3 we describe the different maser transitions of $\mathrm{SiO}, \mathrm{H}_{2} \mathrm{O}$ and $\mathrm{HCN}$ that can be detected in the ALMA frequency range. The results of our models for each molecular species are detailed in Sect. 4. In Sect. 5 we give an estimate of the polarized fluxes that can be expected when observing with ALMA.

\section{Maser polarization theory}

Polarized maser radiation provides a unique tool for studying the role of magnetic fields inside high-density enviroments such as SFRs and circumstellar envelopes of evolved stars. Although the general maser emission mechanims from astronomical sources have been well understood throughout the past thirty years (e.g. Elitzur 1992, Gray 2012), the generation and radiative transfer of polarized maser emission have been difficult to deal with. The polarization properties of maser emission strongly depend on the radiative conditions of the region where the maser is being generated (saturated or unsaturated) and on the nature of the molecular species generating the maser emission (paramagnetic or non-paramagnetic) (Watson 2008; Dinh-v-Trung 2009).

Maser-polarized radiation can be produced in both unsaturated and saturated frames. Maser emission is considered to be saturated when the rate for the stimulated emission $R$ overcomes the decay rate of the molecular state involved, $\Gamma$. In this case, the growth of the possible polarization modes is determined by the population of the molecular states that can interact with a particular mode of the polarization (Nedoluha \& Watson 1990a). Goldreich et al. (1973) first identified two regimes where polarized emission can be generated in the saturated frame for the molecular rotational transition $J=1-0$ : a) The strong magnetic field strength regime, where the Zeeman frequency $g \Omega \gg R$ and $g \Omega \gg \Gamma$; and b) The intermediate magnetic field strength regime, where $(g \Omega)^{2} / \Gamma \gg R \gg g \Omega$. Western \& Watson (1984), Deguchi \& Watson (1990) and Nedoluha \& Watson (1990a) extended the treatment of Goldreich et al. (1973), solving the radiative transfer equations for polarized radiation as a function of the emerging intensity $R / \Gamma$, i.e. as a function of the saturation level. They considered linear, non-paramagnetic molecules, including the rotational transitions $J=2-1$ and $J=3-2$. Nedoluha \& Watson (1990a) have shown that in the presence of a plausible magnetic field and without differences in the population of the magnetic substates involved in the maser emission, the upper limit of fractional linear polarization $p_{L}$ that maser transitions other than $J=1-0$ can achieve is $33 \%$. The fractional linear polarization decreases for transitions with higher angular momentum, and is a function of the angle $\theta$ between the magnetic field $(\boldsymbol{B})$ and the direction of the maser radiation $(\boldsymbol{k})$.

Molecular states with angular momentum higher than $J=$ $1-0$ reach their maximum $p_{L}$ value when the magnetic field lines are perpendicular to the direction of the propagation of the maser radiation, and have $p_{L}=0$ when $\theta$ has the critical value $\theta_{\mathrm{cr}} \sim$ $55^{\circ}$, also known as the van Vleck angle, and when $\theta=0^{\circ}$.

The angle $\phi$ between the polarization vector and the plane $\boldsymbol{k} \cdot \boldsymbol{B}$ also varies as a function of the ratio $g \Omega / R$. For a fixed $\theta$, the vector of polarization should be either perpendicular or parallel to the $\boldsymbol{k} \cdot \boldsymbol{B}$ plane if the condition $g \Omega \gg R$ is satisfied. On the other hand, for higher values of $R$, but still in the intermediate-strength magnetic field regime, the vector of polarization is already rotated away from the $\boldsymbol{k} \cdot \boldsymbol{B}$ plane, i.e. if $R>g \Omega$ the vector of polarization is neither parallel nor perpendicular to the plane $\boldsymbol{k} \cdot \boldsymbol{B}$. In this context, the van Vleck angle is the limit for $\theta$ where the polarization vector changes from being parallel to be perpendicular to the $\boldsymbol{k} \cdot \boldsymbol{B}$ plane. Therefore, as long as the conditions $g \Omega>R$ and $g \Omega>\Gamma$ are satisfied, the information about the morphology of the magnetic field can be extracted from the polarization vector.

Observationally, fractional linear polarization of up to $100 \%$ for $J=1-0 \mathrm{SiO}$ maser transitions (Amiri et al. 2012) and values higher than $33 \%$ for $\mathrm{SiO}$ molecular transitions involving higher angular momentum states (e.g Vlemmings et al. 2011) have been detected. Anisotropic pumping seems to be a possible process to explain such high $p_{L}$ levels (Nedoluha \& Watson 1990a). It can be produced by differences in the angular distribution of the radiation field involved in the population inversion process. In the absence of a magnetic field, anisotropic pumping could produce highly linearly polarized emission. In contrast to the effect of a magnetic field alone in the maser region, the fractional linear polarization can increase with the angular momentum due to an anisotropic population of the involved magnetic substates. Nevertheless, even considering that the linear polarization has been enhanced by anisotropic pumping, if $g \Omega>R$ and $g \Omega>\Gamma$ for the detected line, the magnetic field structure can still be traced directly from the polarization vector, because the magnetic field is the dominant axis of symmetry (Watson 2002). The study of magnetic fields from maser linear polarization thus depends critically on an analysis of the Zeeman frequency $g \Omega$ and the stimulated emission rate $R$.

\section{Observations of (sub)millimetre masers}

\section{1. $\mathrm{SiO}$}

Although it was first detected towards the Orion molecular cloud (Snyder \& Buhl 1974), later surveys have shown that SiO maser emission is quite uncommon in SFRs (Zapata et al. 2009). To date, the only submillimetre $\mathrm{SiO}$ masers detected in SFRs are the $v=1, J=1-0, J=3-2$ and the $v=2, J=1-0$ rotational transitions (Buhl et al. 1974; Davis et al. 1974). In contrast, strong $\mathrm{SiO}$ emission arises from the innermost regions of the CSE of late-type stars, and a number of maser transitions have been detected in vibrational levels of up to $v=4$, with 
A. F. Pérez-Sánchez and W. H. T. Vlemmings: Linear polarization of submillimetre masers

Table 1. Spontaneous emission coefficient and frequencies of the detected $\mathrm{SiO}$ maser transitions in the ALMA frequency range.

\begin{tabular}{|c|c|c|c|c|c|c|c|c|c|}
\hline \multicolumn{5}{|c|}{${ }^{28} \mathrm{SiO}$} & \multicolumn{5}{|c|}{${ }^{29} \mathrm{SiO}$} \\
\hline$v$ & $J_{u}-J_{d}$ & $\begin{array}{c}\text { Freq } \\
(\mathrm{GHz})\end{array}$ & $\begin{array}{c}A \\
\left(\mathrm{~s}^{-1}\right) \\
\end{array}$ & $\begin{array}{c}\text { ALMA } \\
\text { band }\end{array}$ & $v$ & $J_{u}-J_{d}$ & $\begin{array}{c}\text { Freq } \\
(\mathrm{GHz})\end{array}$ & $\begin{array}{c}A \\
\left(\mathrm{~s}^{-1}\right) \\
\end{array}$ & $\begin{array}{c}\text { ALMA } \\
\text { band }\end{array}$ \\
\hline \multirow[t]{2}{*}{0} & $1-0$ & 43.42386 & $3.036 \times 10^{-6}$ & 1 & \multirow{6}{*}{0} & $1-0$ & 42.87992 & $2.114 \times 10^{-8}$ & 1 \\
\hline & $2-1$ & 86.84699 & $2.915 \times 10^{-5}$ & 2 & & $2-1$ & 85.75906 & $2.460 \times 10^{-7}$ & 2 \\
\hline \multirow{7}{*}{1} & $1-0$ & 43.12208 & $3.011 \times 10^{-6}$ & 1 & & $3-2$ & 128.63685 & $9.696 \times 10^{-7}$ & 4 \\
\hline & $2-1$ & 86.24344 & $2.891 \times 10^{-5}$ & 2 & & $4-3$ & 171.51255 & $2.532 \times 10^{-6}$ & 5 \\
\hline & $3-2$ & 129.36326 & $1.045 \times 10^{-4}$ & 4 & & $5-4$ & 214.38548 & $5.334 \times 10^{-6}$ & 6 \\
\hline & $4-3$ & 172.48102 & $2.569 \times 10^{-4}$ & 5 & & $6-5$ & 257.25493 & $9.869 \times 10^{-6}$ & 6 \\
\hline & $5-4$ & 215.59592 & $5.131 \times 10^{-4}$ & 6 & \multirow{3}{*}{1} & $3-2$ & 127.74849 & $3.350 \times 10^{-4}$ & 4 \\
\hline & $6-5$ & 258.70725 & $9.003 \times 10^{-4}$ & 6 & & $4-3$ & 170.32807 & $8.745 \times 10^{-4}$ & 5 \\
\hline & $7-6$ & 301.81433 & $1.445 \times 10^{-3}$ & 7 & & $6-5$ & 255.47849 & $3.407 \times 10^{-3}$ & 6 \\
\hline \multirow{7}{*}{2} & $1-0$ & 42.82059 & $2.986 \times 10^{-6}$ & 1 & 2 & $6-5$ & 253.70317 & 1.11139 & 6 \\
\hline & $2-1$ & 85.64046 & $2.866 \times 10^{-5}$ & 3 & \multirow{2}{*}{\multicolumn{5}{|c|}{${ }^{30} \mathrm{SiO}$}} \\
\hline & $3-2$ & 128.45881 & $1.036 \times 10^{-4}$ & 4 & & & & & \\
\hline & $4-3$ & 171.27507 & $2.547 \times 10^{-4}$ & 5 & \multirow{3}{*}{0} & $1-0$ & 42.373426 & $2.016 \times 10^{-8}$ & 1 \\
\hline & $5-4$ & 214.08848 & $5.088 \times 10^{-4}$ & 6 & & $2-1$ & 84.746170 & $2.346 \times 10^{-7}$ & 2 \\
\hline & $6-5$ & 256.89831 & $8.927 \times 10^{-4}$ & 6 & & $5-4$ & 211.853473 & $5.081 \times 10^{-6}$ & 6 \\
\hline & $7-6$ & 299.70386 & $1.433 \times 10^{-3}$ & 7 & 1 & $4-3$ & 168.323352 & $8.054 \times 10^{-4}$ & 5 \\
\hline \multirow{4}{*}{3} & $1-0$ & 42.51938 & $2.951 \times 10^{-6}$ & 1 & 2 & $4-3$ & 167.160563 & $2.542 \times 10^{-1}$ & 5 \\
\hline & $3-2$ & 127.55521 & $1.027 \times 10^{-4}$ & 4 & & & & & \\
\hline & $4-3$ & 170.07057 & $2.525 \times 10^{-4}$ & 5 & & & & & \\
\hline & $5-4$ & 212.58248 & $5.044 \times 10^{-4}$ & 6 & & & & & \\
\hline 4 & $5-4$ & 211.07784 & $4.986 \times 10^{-4}$ & 6 & & & & & \\
\hline
\end{tabular}

rotational transitions as high as $J=8-7$ (Menten et al. 2006, Humphreys 2007 and references therein). Interferometric obervations have revealed that the $\mathrm{SiO}$ maser emitting regions form ring-like structures centred on the star, between the stellar photosphere and the dust-forming region $\sim 2-6 R_{\star}$ (Diamond et al. 1994; Boboltz \& Diamond 2005). The most common SiO maser transitions detected are the two lowest rotational transitions of the $v=1$ vibrational level, peaking around $43 \mathrm{GHz}$ and $86 \mathrm{GHz}$ (e.g. Barvainis \& Predmore 1985). The list of $\mathrm{SiO}$ rotational transitions, including the isotopologues ${ }^{29} \mathrm{SiO}$ and ${ }^{30} \mathrm{SiO}$, which can be observed as maser emission in the ALMA frequency range, are listed in Table 1 (Müller et al. 2001).

Polarized $\mathrm{SiO}$ maser emission has been detected towards oxygen-rich (post-)AGB stars. Barvainis \& Predmore (1985) reported the detection of strong polarized emission in the rotational transitions $J=1-0$ and $J=2-1$, of both $v=1$ and $v=2$ vibrational levels.

Fractional linear polarization levels between $15 \%$ and $40 \%$ are commonly detected, though levels approaching $100 \%$ have also been reported (e.g. Barvainis \& Predmore 1985; Kemball \& Diamond 1997; Amiri et al. 2012). Shinnaga et al. (2004) and Vlemmings et al. (2011) reported the detection of high fractional linear polarization levels $\left(p_{L} \geq 40 \%\right)$ of the $J=5-4$ rotational transitions of the $v=1$ vibrational level, towards the supergiant VX Sgr. Such high polarization levels cannot only be explained by the presence of a large-scale magnetic field permeating the masing region, but need to be enhanced by non-Zeeman effects.

\section{2. $\mathrm{H}_{2} \mathrm{O}$}

Water is among the most abundant molecules in the envelopes of cores embedded in SFRs and in the CSEs of late-type stars (e.g. Waters et al. 1980; Menten et al. 1990a; Maercker et al. 2008). At submillimetre wavelengths a number of molecular transitions of $\mathrm{H}_{2} \mathrm{O}$ have been detected displaying high flux density values and narrow line shapes characteristic of maser emission lines (Waters et al. 1980; Menten et al. 1990b; Yates $\&$ Cohen 1996). The most studied water maser emission is the low-frequency $6_{16}-5_{23}$ transition at $22.2 \mathrm{GHz}$. Since this transition is not affected by the atmospheric precipitable water vapor (PWV), it has become a reference in the study of water maser emission from astrophysical sources. It has been detected towards the expanding CSEs of late-type stars and high-velocity outflows generated in the envelope of both protostellar objects and (post-) AGB stars. This variety of scenarios gives us an idea of the broad excitation conditions of the $22.2 \mathrm{GHz}$ maser (highdensity regions $n_{\mathrm{H}_{2}}>10^{8} \mathrm{~cm}^{-3}$ and temperatures $2000 \mathrm{~K}>T>$ 200 K, Neufeld \& Melnick 1991; Humphreys 2007). The submillimetre water maser transitions within the ground-vibrational state have been detected towards CSEs of O-rich late-type stars as well as from low- and high-mass SFRs, either tracing shocked regions or arising from the steadily expanding CSEs (e.g. Ivison et al. 1998; Melnick et al. 1993). The different water transitions observable in the ALMA frequency range within the groundvibrational state and the $v_{2}=1$ bending mode are listed in the Table 2 (Pickett et al. 1998). The rotational maser transitions within the vibrationally excited level $v_{2}=1$ are thought to arise from regions where the physical conditions are similar to those that invert the level population of the $\mathrm{SiO}$ rotational transitions (Alcolea \& Menten 1993).

In contrast to the case of $\mathrm{SiO}$, there are not many references in the literature reporting the detection of $\mathrm{H}_{2} \mathrm{O}$ maser polarized radiation in the ALMA frequency range. To date, only Harwit et al. (2010) have succeeded in measuring linear polarization of water maser radiation at $620.7 \mathrm{GHz}$. Unfortunately, the very low atmospheric transmission around $621 \mathrm{GHz}$ prevents the detection of this maser transition from ground-based telescopes.

The excitation conditions of the submillimetre water masers seem to be a subset of the broad excitation conditions generating the $22.2 \mathrm{GHz}$ maser line (Neufeld \& Melnick 1991; Humphreys 2007). The dominating pumping process depends 
Table 2. Einstein coefficient and frequencies of the detected $\mathrm{H}_{2} \mathrm{O}$ and $\mathrm{HCN}$ maser transitions at submillimetre wavelengths.

\begin{tabular}{|c|c|c|c|c|c|c|c|c|c|}
\hline \multicolumn{5}{|c|}{$\mathrm{H}_{2} \mathrm{O}$} & \multicolumn{5}{|c|}{$\mathrm{HCN}$} \\
\hline & Transition & $\begin{array}{c}\text { Freq } \\
(\mathrm{GHz})\end{array}$ & $\begin{array}{c}A \\
\left(\mathrm{~s}^{-1}\right)\end{array}$ & $\begin{array}{c}\text { ALMA } \\
\text { band }\end{array}$ & & Transition & $\begin{array}{c}\text { Freq } \\
(\mathrm{GHz})\end{array}$ & $\begin{array}{c}A \\
\left(\mathrm{~s}^{-1}\right)\end{array}$ & $\begin{array}{c}\text { ALMA } \\
\text { band }\end{array}$ \\
\hline & $3_{13}-2_{20}$ & 183.31012 & $3.629 \times 10^{-6}$ & 5 & & $1-0$ & 88.631602 & $1.771 \times 10^{-7}$ & $2 / 3$ \\
\hline & $10_{29}-9_{36}$ & 321.22564 & $6.348 \times 10^{-6}$ & 7 & $v_{2}=2^{0}$ & $1-0$ & 89.0877 & $1.483 \times 10^{-4}$ & $2 / 3$ \\
\hline & $5_{15}-4_{22}$ & 325.15292 & $1.166 \times 10^{-5}$ & 7 & \multirow{3}{*}{$v_{2}=1^{1_{c}}$} & $2-1$ & 177.2387 & $4.578 \times 10^{-5}$ & 5 \\
\hline & $17_{413}-16_{710}$ & 354.8089 & $1.096 \times 10^{-5}$ & 7 & & $3-2$ & 267.1993 & $2.262 \times 10^{-4}$ & 6 \\
\hline & $7_{53}-6_{60}$ & 437.34667 & $2.212 \times 10^{-5}$ & 8 & & $4-3$ & 354.4605 & $6.122 \times 10^{-4}$ & 7 \\
\hline & $6_{43}-5_{50}$ & 439.15081 & $2.857 \times 10^{-5}$ & 8 & $v_{2}=4$ & $9-8$ & 804.7509 & $a$ & 10 \\
\hline & $6_{42}-5_{51}$ & 470.88895 & $3.534 \times 10^{-5}$ & 8 & \multirow[t]{5}{*}{$v_{1}=1^{1} \rightarrow v_{2}=4^{0}$} & \multirow[t]{5}{*}{$10-9$} & \multirow[t]{5}{*}{890.761} & \multirow[t]{5}{*}{$a$} & \multirow[t]{5}{*}{10} \\
\hline \multirow{4}{*}{$v_{2}=1$} & $4_{40}-5_{33}$ & 96.26116 & $4.719 \times 10^{-7}$ & 3 & & & & & \\
\hline & $5_{50}-6_{43}$ & 232.68670 & $4.770 \times 10^{-6}$ & 6 & & & & & \\
\hline & $6_{61}-7_{52}$ & 293.6645 & $a$ & 6 & & & & & \\
\hline & $1_{10}-1_{01}$ & 658.00655 & $5.568 \times 10^{-3}$ & 9 & & & & & \\
\hline
\end{tabular}

Notes. ${ }^{(a)}$ No spectroscopic data available.

on the characteristics of the region where the maser emission arises; either from post-shock regions, where the inversion of the population of the molecular states is mainly collisional, or from the steadily expanding CSEs, where the pumping process is mainly thought to be caused by infrared photons of warm dust emission. Observations of $22.2 \mathrm{GHz} \mathrm{H}_{2} \mathrm{O}$ maser polarized emission have been used to probe the magnetic field strengths within the steadily expanding CSEs of AGB and supergiant stars (e.g. Vlemmings et al. 2005). In addition, polarized water maser emission has been detected towards the highvelocity outflows of the so-called water-fountains, with spectral features displaying high levels of fractional linear polarization $\left(p_{L}>5 \%\right)$ and unusually broad velocity ranges. These observations have probed magnetically collimated "jets" that appear during the post-AGB phase, and are thought to be the precursors of bipolar (multipolar) planetary nebulae (Vlemmings et al. 2006a; Pérez-Sánchez et al. 2011).

\section{3. $\mathrm{HCN}$}

Strong maser emission has also been detected from $\mathrm{HCN}$ in the CSEs of several carbon-rich (C-rich) AGB stars in the ALMA frequency range (e.g. Bieging 2001; Schilke \& Menten 2003). Table 2 summarizes the different $\mathrm{HCN}$ transitions that can be observed as maser emission in the submillimetre wavelength regime (Müller et al. 2001). Linearly polarized $\mathrm{HCN}$ maser emission of the $89.087 \mathrm{GHz}, J=1-0$ transition, within the $\left(0,2^{0}, 0\right)$ vibrationally excited state, has been detected at approximately $20 \%$ towards the innermost region of the CSE of the C-rich star CIT 6 (Goldsmith et al. 1988). The masing region is thought to be located between the photosphere and the inner radius of the expanding envelope, similar to the $\mathrm{SiO}$ masers in the oxygen-rich late-type stars. The pumping of the $\mathrm{HCN}$ $J=1-0$ transition is more likely caused by the absorption of infrared photons, not by collisional processes, though a combination of both processes cannot be ruled out (Goldsmith et al. 1988). Anisotropies in the population inversion of the masing transitions might affect the fractional linear polarization, but more observations are needed to determine the exact cause of the polarization.

\section{Model results}

Maser emission can be affected by several non-Zeeman processes that can enhance or even produce linear and/or circular
Table 3. Fixed parameter values assumed in our numerical models.

\begin{tabular}{lccccc}
\hline \hline Molecule & $g$ & $\begin{array}{c}\Gamma \\
\left(\mathrm{s}^{-1}\right)\end{array}$ & $\begin{array}{c}g \Omega_{1 \mathrm{G}}{ }^{a} \\
\left(\mathrm{~s}^{-1}\right)\end{array}$ & $\begin{array}{c}\mathrm{B}^{b} \\
(\mathrm{G})\end{array}$ & $\begin{array}{c}\Delta \Omega \\
(\mathrm{sr})\end{array}$ \\
\hline $\mathrm{SiO}$ & 0.155 & 5 & 1480.96 & 1 & $10^{-2}$ \\
$\mathrm{H}_{2} \mathrm{O}$ & 0.65 & 1 & 6226.22 & 0.05 & $10^{-2}$ \\
$\mathrm{HCN}$ & 0.098 & 1 & 957.8 & 1 & $10^{-2}$ \\
\hline
\end{tabular}

Notes. ${ }^{(a)}$ Values calculated using Eq. (2) for a magnetic field $B=1 \mathrm{G}$. (b) Magnetic field of the same order of magnitude as the values detected towards SFRs and CSE of late-type stars for regions at densities of $n_{\mathrm{H}_{2}}=10^{10} \mathrm{~cm}^{-3}$ in the case of $\mathrm{SiO}$ and $\mathrm{HCN}$, and $n_{\mathrm{H}_{2}}=10^{8} \mathrm{~cm}^{-3}$ in the case of $\mathrm{H}_{2} \mathrm{O}$.

polarization, such as anisotropic pumping or the change of the quantization axis along the amplification path (Wiebe \& Watson 1998). Therefore, it is necessary to solve the radiative transfer equation for a particular rotational transition to learn whether the polarization detected can be correlated to a large-scale magnetic field permeating the masing region. Furthermore, it is necessary to determine if the involved rotational transition can produce appreciable levels of $p_{L}$ when the quantization axis is defined by the magnetic field direction, i.e. when the Zeeman frequency $g \Omega$ is higher than the stimulated emission rate $R$. The inequalities $g \Omega>\Gamma$ and $g \Omega>R$ allow us to analyse the polarization observed in terms of the ordinary population of the magnetic substates (Watson 2008). In the case of $\mathrm{SiO}$ the decay rate $\Gamma$ listed in Table 3 corresponds to the rate for the radiative decay from the first vibrationally excited state to the groundvibrational state (Nedoluha \& Watson 1990a). For water, this value is roughly the inverse lifetime for infrared transitions of the $22 \mathrm{GHz}, \mathrm{H}_{2} \mathrm{O}$ maser transition (Nedoluha \& Watson 1990b). In the case of $\mathrm{HCN}$, this value corresponds to the decay rate associated to a pumping process dominated by infrared radiation (Goldreich \& Kwan 1974). Considering the $\Gamma$ and $g \Omega$ values in Table 3, it is clear that for the case of interest the criterium $g \Omega>\Gamma$ is satisfied. To evaluate whether the stimulated emission rate satisfies the condition $g \Omega>R$ for a particular transition when $p_{L}$ is sufficiently large to be detected, we have used a radiative transfer code adapted from Nedoluha $\&$ Watson (1992) and Vlemmings (2002). The stimulated emission rate $R$ is given by

$R \approx \frac{A k T_{\mathrm{b}} \Delta \Omega}{4 \pi h v}$ 
where $A$ is the Einstein coefficient of the involved transition, which is calculated and listed for most of the transitions in Tables 1 and 2; $k$ and $h$ are the Boltzmann and Planck constant, $v$ is the maser frequency, $T_{\mathrm{b}}$ is the brightness temperature and $\Delta \Omega$ is the relation between the real angular size of the of the masing cloud and the observed angular size. Although the quantities $T_{\mathrm{b}}$ and $\Delta \Omega$ are related to the observed intensity of the maser, it is a difficult task to constrain their value directly from observations. Brightness temperatures of up to $\sim 10^{15} \mathrm{~K}$ have been measured for water masers in SFRs, whereas estimated values for $\Delta \Omega$ are $\sim 10^{-1}-10^{-2}$ sr for water masers detected towards the CSEs of late-type stars (Richards et al. 2011; Vlemmings \& van Langevelde 2005), and in some cases $\Delta \Omega \sim 10^{-5} \mathrm{sr}$ for the masers detected towards SFRs (Nedoluha \& Watson 1991).

The fractional linear polarization increases with $T_{\mathrm{b}} \Delta \Omega$ in the presence of a magnetic field as long as $\theta \neq \theta_{\mathrm{cr}}$. Since $R$ increases faster than $p_{L}$ when $T_{\mathrm{b}} \Delta \Omega$ increases, the polarization vector of linearly polarized maser radiation detected with high brightness temperature does not necessarily satisfy the criterium $g \Omega>R$, and thus cannot always be directly correlated with the direction of the magnetic field lines, unless the emitted radiation has a high degree of beaming. On the other hand, low brightness temperatures might result in very low or undetectable values of fractional linear polarization. Furthermore, the value of $T_{\mathrm{b}} \Delta \Omega$ scales with the ratio $R / \Gamma$, and higher values of the molecular decay rate $\Gamma$ imply a lower level of fractional linear polarization for the same $T_{\mathrm{b}} \Delta \Omega$ value. This reflects the dependence of the fractional linear polarization level of a particular rotational transition on the saturation level of the maser radiation.

The Zeeman frequency $g \Omega$ determines the energy-splitting of the magnetic sub-levels. In a masing region permeated by a constant magnetic field parallel to the $z$-axis of the coordinate system, the energy-shifting of the magnetic sub-levels is given by $\hbar g \Omega m / 2=g \mu_{N} B m$, where $\mu_{N}$ and $m$ are the nuclear magneton and the quantum number of the magnetic substate (Nedoluha $\&$ Watson 1990a). Hence the values for $g \Omega$ can be estimated using the relation

$g \Omega=\frac{2 g \mu_{N}}{\hbar} \frac{B[\mathrm{G}]}{1 \times 10^{4}}$,

where the factor $1 \times 10^{4}$ originates from the conversion between the units of Tesla and Gauss. In general, the molecular Landé factor $(g)$ is different for each magnetic sub-level. The data in the literature are very limited, and there are no reported $g$-factor values for all the different rotational transitions of $\mathrm{HCN}$ nor $\mathrm{H}_{2} \mathrm{O}$. The Landé $g$-factor for $\mathrm{SiO}$ has minor differences (less than $1 \%$ ) for the $v=0, v=1$ and $v=2$ vibrational transitions (Landolt-Börnstein 1982). The assumed $g$-factors for the different molecules are listed in Table 3. In the case of $\mathrm{H}_{2} \mathrm{O}$, Nedoluha \& Watson (1992) calculated the $g \Omega$ values considering hyperfine splitting for the $6_{16}$ and $5_{23}$ rotational states of water. Nevertheless, in the present case, we have assume the lower limit suggested for $g$ as it would be without hyperfine splitting (Kukolich 1969), to calculate $g \Omega$ for the different $\mathrm{H}_{2} \mathrm{O}$ rotational transitions that we modelled. For $\mathrm{HCN}$ we assumed the lower $g$ factor value reported for the $\left(0,1^{1_{c}}, 0\right)$ vibrational state, which corresponds to a magnetic field parallel to the molecular symmetry axis (Goldsmith et al. 1988 and references therein). Because for closed-shell, non-paramagnetic molecules the response to a magnetic field is weak, we consider the $g$-factor values listed in Table 3 as a conservative choice to constrain a minimum value for $g \Omega$ for the different molecular species. The $g \Omega$ values presented in Table 3 correspond to $B=1 \mathrm{G}$ in Eq. (2), and can be scaled according to magnetic field strengths
Table 4. Model results.

\begin{tabular}{lc|cc|cc}
\hline \hline \multicolumn{2}{c|}{$\mathrm{SiO}$} & \multicolumn{2}{c|}{$\mathrm{H}_{2} \mathrm{O}$} & \multicolumn{2}{c}{$\mathrm{HCN}$} \\
\hline$J_{u}-J_{d}$ & $p_{L}$ & $J_{u}-J_{d}$ & $p_{L}$ & $J_{u}-J_{d}$ & $p_{L}$ \\
\hline $1-0$ & $\sim 0.32$ & $3_{13}-2_{20}$ & $\sim 0.19$ & $1-0$ & $\sim 0.33$ \\
$2-1$ & $\sim 0.23$ & $5_{15}-4_{22}$ & $\sim 0.16$ & $2-1$ & $\sim 0.27$ \\
$3-2$ & $\sim 0.18$ & $6_{43}-5_{50}$ & $\sim 0.15$ & $3-2$ & $\sim 0.22$ \\
$4-3$ & $\sim 0.15$ & & & $4-3$ & $\sim 0.19$ \\
$5-4$ & $\sim 0.14$ & & & & \\
$6-5$ & $\sim 0.13$ & & & & \\
\hline
\end{tabular}

Notes. Values of $p_{L}$ assuming $\theta=90^{\circ}$ and $g \Omega=10 R$ for rotational transitions of the $v=1$ vibrational state of $\mathrm{SiO}$ and the ground-vibrational level of $\mathrm{H}_{2} \mathrm{O}$. For $\mathrm{HCN}$, the $J=1-0$ belongs to the vibrationally excited $v_{2}=2^{\circ}$ state, whereas the higher $J$ transitions listed are from the $v_{2}=1^{1_{c}}$ vibrationally excited level.

reported for the different masing regions in both SFRs and CSEs of (post-)AGB stars.

Single-dish observations have revealed average magnetic field strengths of $\sim 3.5 \mathrm{G}$ in the $\mathrm{SiO}$ maser region, whereas for $\mathrm{H}_{2} \mathrm{O}$ the measured values range between $100-300 \mathrm{mG}$ for the CSEs of AGB and supergiant stars, and between $15-150 \mathrm{mG}$ at densities of $n_{\mathrm{H}_{2}}=10^{8}-10^{11} \mathrm{~cm}^{-3}$ in SFRs. For our models we assumed the values of the magnetic field strength listed in Table 3. This allows us to analyse our results as a function of the minimum $g \Omega$ values for the three molecular species modelled. Thus, using Eq. (2) for the corresponding magnetic field in Table 3, we calculate the $g \Omega$ in order to determine whether the inequality $g \Omega>R$ is satisfied or not.

\section{Analysis}

\subsection{SiO maser}

We present the results of our models for the SiO rotational transitions $J=5-4$ and $J=2-1$ of the $v=1$ vibrational state in Fig. 1. The results are for four different $\theta$ values and the vertical line corresponds to a $T_{\mathrm{b}} \Delta \Omega$ where $g \Omega=10 R$. The ideal case of $\theta=90^{\circ}$ determines the maximum fractional linear polarization value when a magnetic field permeates the masing region. The maximum fractional linear polarization for rotational transitions of the first $\mathrm{SiO}$ vibrationally excited state are listed in Table 4. These upper limits were established using the corresponding $g \Omega$ value listed in Table 3 for each molecular species, and subsequently finding the $T_{\mathrm{b}} \Delta \Omega$ values where $g \Omega=10 R$. According to our results, it is possible to generate $p_{L}$ values of up to $13 \%$ for the $J=5-4$ rotational transition, and of up to $23 \%$ for the $J=2-1$ rotational transition without consideration of non-Zeeman effects (Fig. 1), while the much higher values of $p_{L}$ detected for the $\mathrm{SiO}$ masers require anisotropic pumping.

Therefore, although anisotropic pumping has a strong impact on the fractional linear polarization level, submillimetre $\mathrm{SiO}$ maser features observed with brightness temperatures $<10^{9}-10^{10} \mathrm{~K}$ fulfil $g \Omega>R$ and could in principle be used to trace the structure of the magnetic field permeating the $\mathrm{SiO}$ masing region.

\section{2. $\mathrm{H}_{2} \mathrm{O}$ maser}

To determine the level of fractional linear polarization that water maser transitions can reach when the masing region is permeated by a large-scale magnetic field, we ran numerical models of the rotational transitions within the ground-vibrational state, 
$215.59 \mathrm{GHz} \mathrm{J}=5-4 \mathrm{SiO} \mathrm{v}=1$

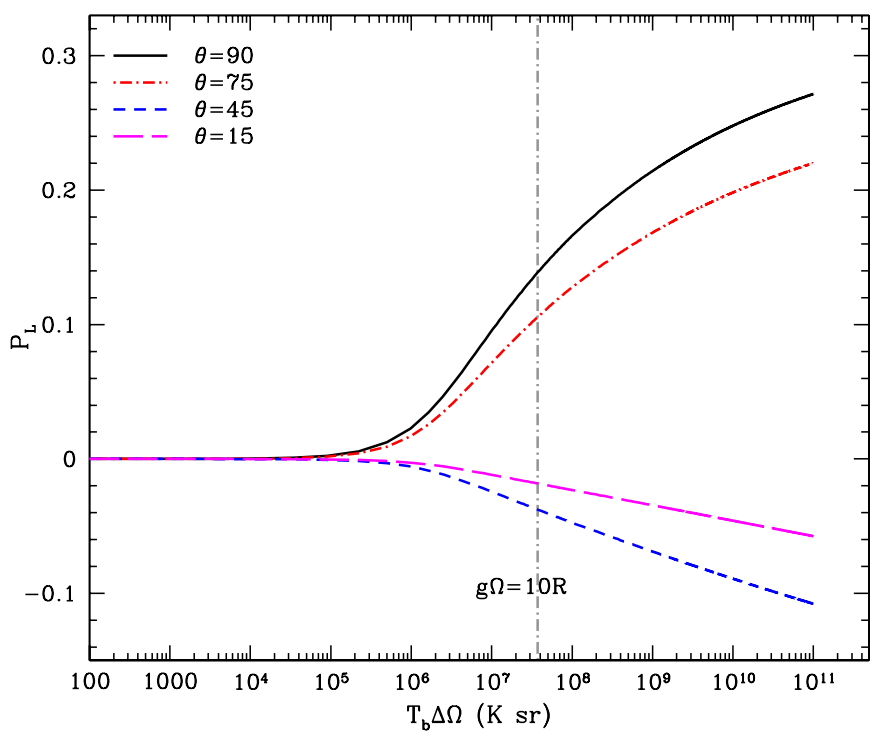

86.243 $\mathrm{GHz} \mathrm{J}=2-1 \mathrm{SiO} \mathrm{v}=1$

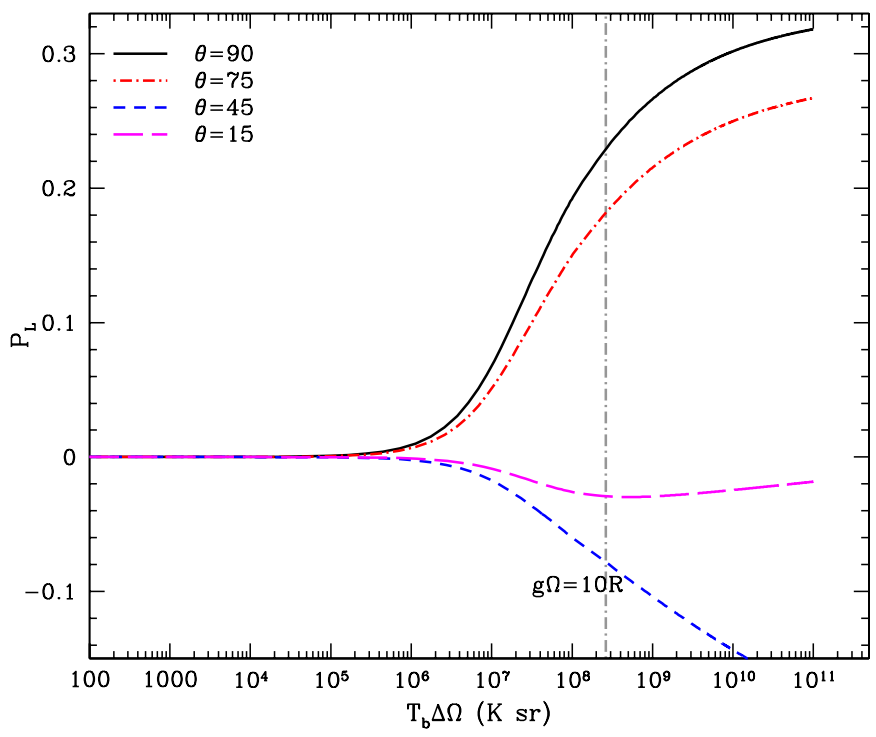

Fig. 1. Fractional linear polarization as a function of $T_{\mathrm{b}} \Delta \Omega$ for the $215 \mathrm{GHz}, J=5-4$ (top), and the $86.2 \mathrm{GHz}, J=2-1$ (bottom) $\mathrm{SiO}$ rotational transitions within the $v=1$ vibrational level for four values of the angle between the magnetic field lines and the direction of propagation of the maser radition, $\theta$, using the parameters listed in Table 3 for $\mathrm{SiO}$. Positive and negative $p_{L}$ values mean that the vector of polarization is either perpendicular or parallel the magnetic field lines projected on the plane of the sky. The vertical line sets the $T_{\mathrm{b}} \Delta \Omega$ value such that $g \Omega=10 R$.

using the assumptions described in Sect. 5.1, together with the corresponding parameters in Table 3 . The results are presented in Table 4. In Fig. 2 we present the results for the $183 \mathrm{GHz}$ and $325 \mathrm{GHz}$ lines for four different $\theta$ values. Our results suggest upper limits for fractional linear polarization of up to $19 \%$ and $16 \%$ for the $183 \mathrm{GHz}$ and $325 \mathrm{GHz}$ lines, respectively. Therefore, considering the values we assumed for the input parameters listed in Table 3, maser features with $10^{8} \mathrm{~K}<T_{\mathrm{b}}<$ $10^{11} \mathrm{~K}$ can produce observable fractional linear polarization levels that can be used to determine the magnetic field morphology. However, if $T_{\mathrm{b}}$ is larger, the observed polarization direction will
$183 \mathrm{GHz} 3_{13}-2_{20} \mathrm{H}_{2} \mathrm{O}$

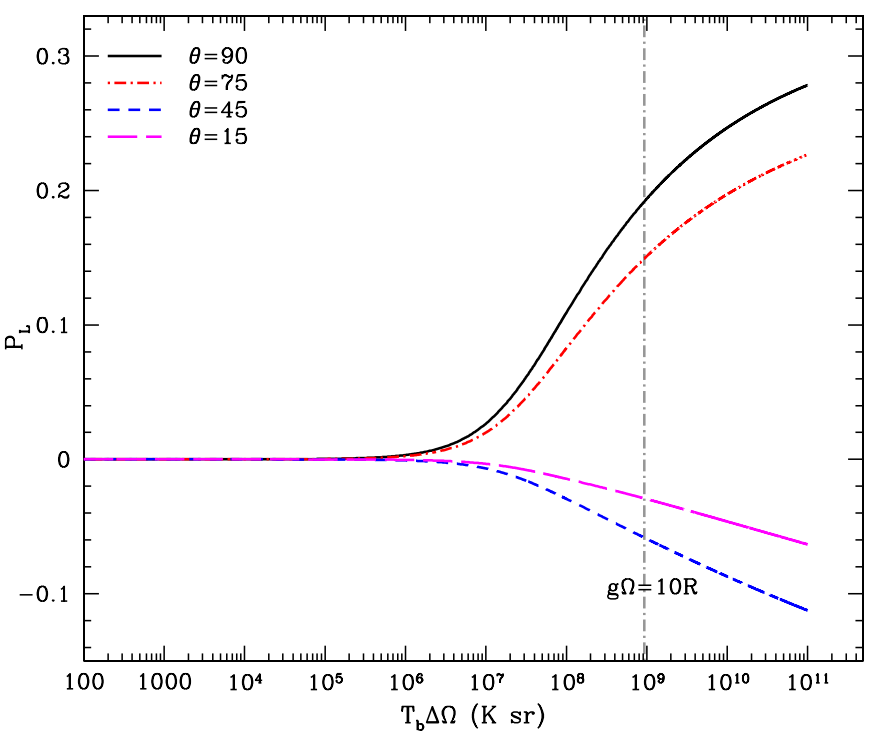

$325 \mathrm{GHz} 5_{15}-4_{22} \mathrm{H}_{2} \mathrm{O}$

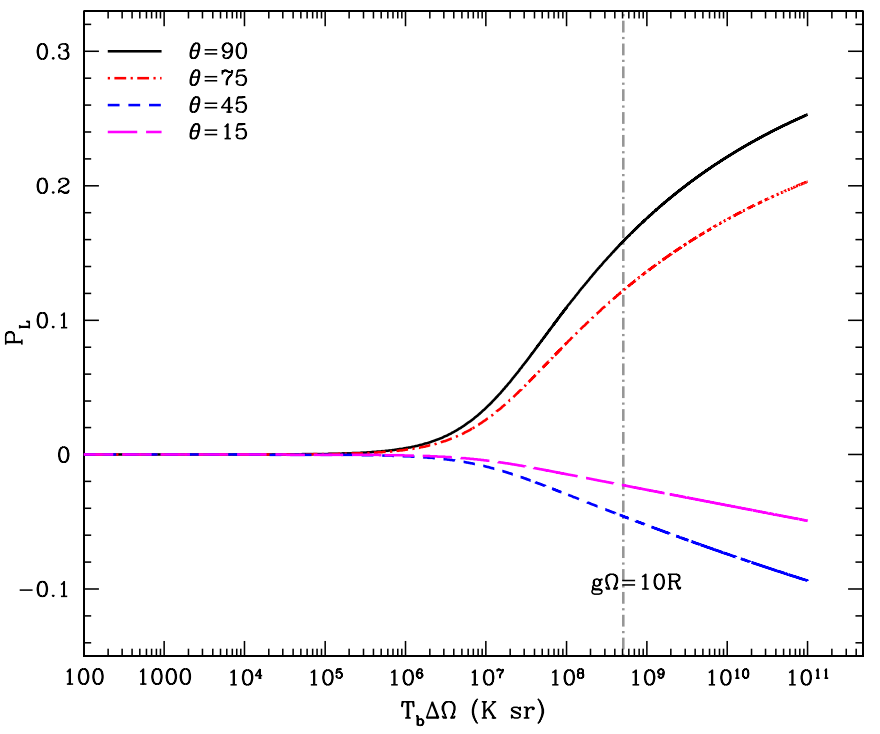

Fig. 2. Fractional linear polarization as a function of $T_{\mathrm{b}} \Delta \Omega$ for the $183 \mathrm{GHz}, 3_{13}-2_{20}$ (top), and the $325 \mathrm{GHz}, 5_{15}-4_{22}$ (bottom) $\mathrm{H}_{2} \mathrm{O}$ rotational transitions, both within the ground-vibrational level, for four values of the angle between the magnetic field lines and the direction of propagation of the maser radiation, $\theta$. We considered the magnetic field strength and decay rate listed in Table 3 for $\mathrm{H}_{2} \mathrm{O}$. Positive and negative $p_{L}$ values mean that the vector of polarization is either perpendicular or parallel the magnetic field lines projected on the plane of the sky. The vertical line sets the $T_{\mathrm{b}} \Delta \Omega$ value such that $g \Omega=10 R$.

be rotated away from the projected magnetic field direction on the plane of the sky.

\subsection{HCN maser}

In Table 4 we present our results for the maximum fractional linear polarization that the $v_{2}=2^{0}, J=1-0$ rotational transition and higher $J$ transitions within the $v_{2}=1^{1_{c}}$ vibrational state can produce. In Fig. 3 we show our results assuming a magnetic field oriented perpedicular to the direction of propagation of the maser radiation, and the parameters listed in Table 3 for HCN. 


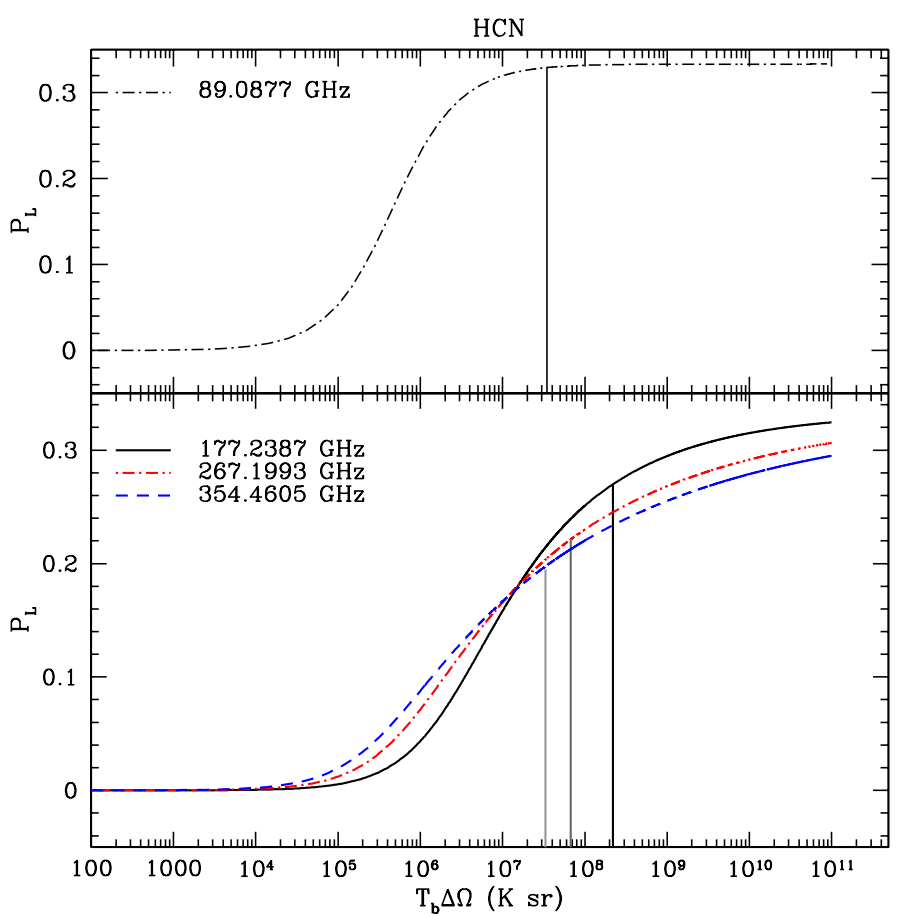

Fig. 3. Fractional linear polarization as a function of $T_{\mathrm{b}} \Delta \Omega$ for $J=1-0$, $v_{1}=2^{0}$ rotational transition (top), and three $\mathrm{HCN}$ rotational transitions of the vibrationally excited state $v_{1}=1^{1_{c}}$ (bottom), assuming a magnetic field oriented perpendicular to the direction of propagation of the maser radiation. We considered the magnetic field strength and decay rate listed in Table 3 for HCN. Under these assumptions, the vertical lines set the maximum linear polarization that the different maser lines can reach satisfying the condition $g \Omega=10 R$.

Fractional linear polarization of up to $\sim 33 \%$ can be generated in the case of the $J=1-0$ rotational transition for $g \Omega=10 R$. This value decreases for rotational transitions with higher $J$ values, as expected. The values of $T_{\mathrm{b}} \Omega$ scale with $R / \Gamma$, and consequently, lower $\Gamma$ values will increase the fractional polarization level that a particular rotational transition can reach while still satisfying $g \Omega>R$. Nevertheless, according to our results, HCN maser emission towards C-rich AGB stars can be generated with high fractional polarization values within the $g \Omega>R$ regime. Thus, the vector of polarization detected from HCN maser observations could be used to probe the magnetic field structure around C-rich AGB stars, even if the level of polarization has been enhanced by non-Zeeman effects.

\section{Observing maser polarization with ALMA}

The temperature and density conditions that favour the population inversion of different masing molecules in SFRs and CSEs of (post-) AGB stars are not the same. Most problably these conditions also differ for different rotational transitions. Therefore, detecting polarized maser emission from multiple rotational transitions and from multiple molecular species with ALMA will enable us to trace the magnetic field structure throughout extended regions around those sources. The accuracy of the ALMA polarimetry will allow us to detect polarized emission of $0.1 \%$ of the detected Stokes $I$. Therefore, fractional linear polarization can be detected in short observations with very good signal-tonoise ratios. To probe whether the measured polarization of a maser traces the magnetic field permeating the masing region, it
Table 5. Sensitivity values at the different maser frequencies of $\mathrm{SiO}$, $\mathrm{H}_{2} \mathrm{O}$ and $\mathrm{HCN}$ within the ALMA frequency range.

\begin{tabular}{cc|cc|cc}
\hline \hline \multicolumn{2}{c}{$\mathrm{SiO}$} & \multicolumn{2}{c}{$\mathrm{H}_{2} \mathrm{O}$} & \multicolumn{2}{c}{$\mathrm{HCN}$} \\
\hline $\begin{array}{c}v \\
(\mathrm{GHz})\end{array}$ & $\begin{array}{c}\sigma \\
(\mathrm{mJy})\end{array}$ & $\begin{array}{c}v \\
(\mathrm{GHz})\end{array}$ & $\begin{array}{c}\sigma \\
(\mathrm{mJy})\end{array}$ & $\begin{array}{c}v \\
(\mathrm{GHz})\end{array}$ & $\begin{array}{c}\sigma \\
(\mathrm{mJy})\end{array}$ \\
\hline 86.243 & 4.58 & 183.310 & 38.37 & 89.0877 & 4.49 \\
129.363 & 4.32 & 325.153 & 39.58 & 177.238 & 4.97 \\
172.481 & 4.74 & 439.151 & 59.25 & 267.199 & 4.12 \\
215.596 & 3.63 & & & 354.461 & 4.99 \\
258.707 & 4.76 & & & & \\
\hline
\end{tabular}

Notes. The $\sigma$ listed corresponds to the sensitivity achieved after $1 \mathrm{~h}$ of on-source time, with $0.1 \mathrm{~km} \mathrm{~s}^{-1}$ for a source that reaches a maximum elevation of $59^{\circ}$.

is necessary to calculate the brightness temperature of the spectral feature and evaluate if the product $T_{\mathrm{b}} \Delta \Omega$ satisfies the inequality $g \Omega>R$ for the observed emission. For detected spectral features it is possible to constrain $T_{\mathrm{b}}$ by considering the equation

$$
\frac{T_{\mathrm{b}}}{[\mathrm{K}]}=\frac{S(v)}{[\mathrm{Jy}]}\left(\frac{\Sigma^{2}}{\left[\mathrm{mas}^{2}\right]}\right)^{-1} \zeta_{v},
$$

where $S(v)$ is the detected flux density, $\Sigma$ is the maser angular size and $\zeta_{v}$ is a constant factor that includes a proportionality factor obtained for a Gaussian shape (Burns et al. 1979). It scales with frequency according to the relation

$\zeta_{v}=6.1305 \times 10^{11}\left(\frac{v}{\mathrm{GHz}}\right)^{-2} \frac{\mathrm{mas}^{2}}{\mathrm{Jy}} \mathrm{K}$.

We calculated the rms value at the different maser frequencies that we modelled using the ALMA sensitivity calculator for an array of $5012-\mathrm{m}$ antennas. The results are presented in Table 5 and correspond to observations of $1 \mathrm{~h}$ of on-source time with $0.1 \mathrm{~km} \mathrm{~s}^{-1}$ of spectral resolution. To estimate the brightness temperature from observations that do not resolve individual features, it is necessary to assume a value for the size of the masing region generating the spectral feature. VLBI studies have revealed masers to be very compact spots with typical sizes of $1 \mathrm{AU}$. Based on observed peak-flux density values for maser emission of $\mathrm{SiO}, \mathrm{H}_{2} \mathrm{O}$ and $\mathrm{HCN}$, assuming a size of 1 mas, i.e. a source distance of $1 \mathrm{kpc}$, we here give a few examples of potential ALMA observations of maser polarization.

$\mathrm{SiO}$ masers: $\mathrm{SiO}$ maser emission has been reported displaying flux density values between 7.4 Jy and 64 Jy (Shinnaga et al. 2004) for the $v=1, J=5-4$ rotational transition. Moreover, Barvainis \& Predmore (1985) reported flux density values between $210 \mathrm{Jy}$ and $625 \mathrm{Jy}$ for the $\mathrm{SiO} J=2-1$ rotational transition of the same vibrationally excited state. As an example, we investigate the detection of a maser spectral feature with a peakflux density of $10 \mathrm{Jy}$ of the $\mathrm{SiO} J=5-4(v=1)$ rotational transition. For these values, Eq. (3) gives $T_{\mathrm{b}}=1.32 \times 10^{8} \mathrm{~K}$. Comparing this with the $T_{\mathrm{b}} \Delta \Omega$ value, which corresponds to the limit of $g \Omega=10 R$ (Fig. 1), maser emission with beaming angles $\Delta \Omega<2.84 \times 10^{-1}$ sr will have a linear polarization that can be used to trace the magnetic field direction. But usually $\mathrm{SiO}$ maser spectra exhibit blended components. If this is the case, the flux density observed corresponds to a number of maser spots with similar line-of-sight velocities increasing the observed $S(v)$. Therefore, the brightness temperature derived using Eq. (3) could be overstimated, but still could be used to set an 
upper limit value for $T_{\mathrm{b}}$. To reach the regime where $g \Omega>R$, it is necessary to associate a $\Delta \Omega$ value to the brightness temperature derived from the $S(v)$ of the blended components. Hence, since individual maser spectral features might have lower $T_{\mathrm{b}}$ than the blended feature which contains it, even unbeamed maser radiation could still place the linear polarization detected within the regime where $g \Omega>R$ is satisfied.

Finally, according to the ALMA sensitivity calculator, a $5 \sigma$ detection of $1 \%$ of fractional linear polarization of $\mathrm{SiO}$ maser emission with low peak-flux densities (few tens of Jy/beam) requires short on-source observation time $(t<1 \mathrm{~h})$.

$\mathrm{H}_{2} \mathrm{O}$ masers: Submillimetre $\mathrm{H}_{2} \mathrm{O}$ lines have been detected with flux densities from a few tens to several thousands of Jy. For the minimun peak-flux density (243 Jy/beam) reported for the $183 \mathrm{GHz}$ by van Kempen et al. (2009), the brightness temperature we obtain using Eq. (3) is $T_{\mathrm{b}}=4.43 \times 10^{9} \mathrm{~K}$. Hence, $\mathrm{H}_{2} \mathrm{O}$ maser emission detected with beaming solid angles $\Delta \Omega \geq$ $1.6 \times 10^{-3} \mathrm{sr}$ can generate fractional linear polarization levels higher than $1 \%$ (Fig. 2), satisfying the condition $g \Omega>R$. The $1 \%$ of linear polarization level of the weakest $183 \mathrm{GHz}$ maser feature detected by van Kempen et al. (2009) can be easily detected in very short integration times. Unfortunately, many $\mathrm{H}_{2} \mathrm{O}$ maser lines are affected by the atmospheric PWV. For maser spectral features with peak-flux densities of the order of $20 \mathrm{Jy} /$ beam at $439.151 \mathrm{GHz}$, for instance, a $3 \sigma$ detection of $1 \%$ will require $\sim 1 \mathrm{~h}$ of on-source observation time.

HCN masers: HCN $J=1-0,\left(v_{2}=2^{0}\right)$ maser emission at $89.0877 \mathrm{GHz}$ has been detected with fractional linear polarization of $20 \%$ for a spectral feature with flux density of $38 \mathrm{Jy}$ (Goldsmith et al. 1988). Furthermore, Lucas \& Cernicharo (1989) reported the detection of $\mathrm{HCN} v_{2}=1^{1_{c}}$ maser emission with a peak-flux density $\sim 400 \mathrm{Jy}$ at $177 \mathrm{GHz}$. Schilke et al. (2000) reported the detection of the vibrationally excited $\mathrm{HCN}\left(04^{0} 0\right) J=9-8$ maser line near $805 \mathrm{GHz}$ with a flux density of $\sim 1500 \mathrm{Jy}$.

For emission reported by Goldsmith et al. (1988), assuming a magnetic field perpendicular to the propagation of the maser radiation, $20 \%$ of fractional linear polarization corresponds to $T_{\mathrm{b}} \Delta \Omega=7.65 \times 10^{5} \mathrm{~K} \mathrm{sr}$ (Fig. 3, top), which places the polarization detected in the regime where $g \Omega>R$. On the other hand, considering a spectral feature of $S(v)=38 \mathrm{Jy}$, Eq. (3) gives $T_{\mathrm{b}}=2.94 \times 10^{9} \mathrm{~K}$, implying a beaming angle of $2.6 \times 10^{-4} \mathrm{sr}$, assuming a maser of 1 mas. But still, a beaming angle $\Delta \Omega \sim 10^{-2}$ sr is needed for this maser to satisfy $g \Omega \geq 10 R$ and be a realiable tracer of the magnetic field within the masing region. However, if the emission consists of a contribution from multiple maser spots, the brightness temperature of the spectral feature will be overstimated, as discussed previously for blended $\mathrm{SiO}$ maser lines. Finally, as in the case of $\mathrm{SiO}$ maser emission, $1 \%$ of linear polarization could be observed with very low rms values in short on-source observation times (Table 5).

\section{Conclusions}

We have run numerical models to calculate the fractional linear polarization $p_{L}$ of maser emission generated by the interaction of a magnetic field with the different rotational transitions of $\mathrm{SiO}$, $\mathrm{H}_{2} \mathrm{O}$ and $\mathrm{HCN}$ within the ALMA frequency range. The fractional linear polarization was calculated as a function of $T_{\mathrm{b}} \Delta \Omega$, a quantity that can be related to the stimulated emission rate $R$ of the involved transition. Considering both the minimun value of the Landé $g$-factor for each molecular species and a suitable magnetic field strength for the different masing regions, we found the maximum $p_{L}$ that the analysed rotational transitions can reach while satisfying both conditions $g \Omega>R$ and $g \Omega>\Gamma$. Meeting these criteria allowed us to use the detected vector of polarization as a tracer of the magnetic field in the masing region, even if the polarization observed has been affected by nonZeeman effects.

According to our results, $\mathrm{SiO}, \mathrm{H}_{2} \mathrm{O}$ and $\mathrm{HCN}$ submillimetre maser emission can be detected with observable fractional polarization levels $(>1 \%)$ in the regime $g \Omega>R$. But especially for the strongest masers, a careful analysis of the brightness temperature is needed to confirm that the maser polarization is still in this regime. Thus, observing with ALMA full-polarization capabilities will enable us to use polarized maser emission as tracer of the magnetic field structure towards SFRs and CSEs of (post)AGB stars. Depending on the maser spectral features detected, both the brightness temperature and the beaming solid angle can be better constrained, leading to a more accurate determination of the direction of the magnetic field in the masing region.

Acknowledgements. We wish to thank Matthias Maercker for his useful comments on the manuscript. This research was supported by the Deutsche Forschungsgemeinschaft (DFG) through the Emmy Noether Research grant VL 61/3-1, the DFG SFB 956 grant, and the BMBF ASTRONET project ARTIST.

\section{References}

Alcolea, J., \& Menten, K. M. 1993, in Astrophysical Masers, eds. A. W. Clegg, \& G. E. Nedoluha (Berlin: Springer Verlag), Lecture Notes in Physics, 412, 399

Alves, F. O., Vlemmings, W. H. T., Girart, J. M., \& Torrelles, J. M. 2012, A\&A, 542, A 14

Amiri, N., Vlemmings, W., \& van Langevelde, H. J. 2010, A\&A, 509, A26

Amiri, N., Vlemmings, W. H. T., Kemball, A. J., \& van Langevelde, H. J. 2012, A\&A, 538, A136

Barvainis, R., \& Predmore, C. R. 1985, ApJ, 288, 694

Bieging, J. H. 2001, ApJ, 549, L125

Boboltz, D. A., \& Diamond, P. J. 2005, ApJ, 625, 978

Buhl, D., Snyder, L. E., Lovas, F. J., \& Johnson, D. R. 1974, ApJ, 192, L97

Burns, J. O., Owen, F. N., \& Rudnick, L. 1979, AJ, 84, 1683

Davis, J. H., Blair, G. N., van Till, H., \& Thaddeus, P. 1974, ApJ, 190, L117

Deguchi, S., \& Watson, W. D. 1990, ApJ, 354, 649

Diamond, P. J., Kemball, A. J., Junor, W., et al. 1994, ApJ, 430, L61

Dinh-v-Trung 2009, MNRAS, 399, 1495

Elitzur, M. 1992, Astronomical masers (Kluwer Academic Publishers), Astrophys. Space Sci. Lib., 170

Fish, V. L., \& Reid, M. J. 2007a, ApJ, 656, 952

Fish, V. L., \& Reid, M. J. 2007b, ApJ, 670, 1159

Goldreich, P., \& Kwan, J. 1974, ApJ, 190, 27

Goldreich, P., Keeley, D. A., \& Kwan, J. Y. 1973, ApJ, 179, 111

Goldsmith, P. F., Lis, D. C., Omont, A., Guilloteau, S., \& Lucas, R. 1988, ApJ, 333,873

Gray, M. 2012, Maser Sources in Astrophysics (Cambridge, UK: Cambridge University Press)

Harwit, M., Houde, M., Sonnentrucker, P., et al. 2010, A\&A, 521, L51

Herpin, F., Baudry, A., Thum, C., Morris, D., \& Wiesemeyer, H. 2006, A\&A, 450,667

Humphreys, E. M. L. 2007, in IAU Symp. 242, eds. J. M. Chapman, \& W. A. Baan, 471

Ivison, R. J., Yates, J. A., \& Hall, P. J. 1998, MNRAS, 295, 813

Kemball, A. J., \& Diamond, P. J. 1997, ApJ, 481, L111

Kemball, A. J., Diamond, P. J., Gonidakis, I., et al. 2009, ApJ, 698, 1721

Kukolich, S. G. 1969, J. Chem. Phys., 50, 3751

Landolt-Börnstein 1982, in Group II, 14a, eds. K.-H. Hellwege, \& A.M. Hellwege (Berlin: Springer), 750

Leal-Ferreira, M. L., Vlemmings, W. H. T., Diamond, P. J., et al. 2012, A\&A, 540, A42

Lucas, R., \& Cernicharo, J. 1989, A\&A, 218, L20

Maercker, M., Schöier, F. L., Olofsson, H., Bergman, P., \& Ramstedt, S. 2008, A\&A, 479, 779

Melnick, G. J., Menten, K. M., Phillips, T. G., \& Hunter, T. 1993, ApJ, 416, L37 
A. F. Pérez-Sánchez and W. H. T. Vlemmings: Linear polarization of submillimetre masers

Menten, K. M., Melnick, G. J., \& Phillips, T. G. 1990a, ApJ, 350, L41

Menten, K. M., Melnick, G. J., Phillips, T. G., \& Neufeld, D. A. 1990b, ApJ, 363, L27

Menten, K. M., Philipp, S. D., Güsten, R., et al. 2006, A\&A, 454, L107

Müller, H. S. P., Thorwirth, S., Roth, D. A., \& Winnewisser, G. 2001, A\&A, 370, L49

Nedoluha, G. E., \& Watson, W. D. 1990a, ApJ, 354, 660

Nedoluha, G. E., \& Watson, W. D. 1990b, ApJ, 361, L53

Nedoluha, G. E., \& Watson, W. D. 1991, ApJ, 367, L63

Nedoluha, G. E., \& Watson, W. D. 1992, ApJ, 384, 185

Neufeld, D. A., \& Melnick, G. J. 1991, ApJ, 368, 215

Pérez-Sánchez, A. F., Vlemmings, W. H. T., \& Chapman, J. M. 2011, MNRAS, 418, 1402

Pickett, H. M., Poynter, R. L., Cohen, E. A., et al. 1998, J. Quant. Spectr. Radiat. Transf., 60, 883

Richards, A. M. S., Elitzur, M., \& Yates, J. A. 2011, A\&A, 525, A56

Schilke, P., \& Menten, K. M. 2003, ApJ, 583, 446

Schilke, P., Mehringer, D. M., \& Menten, K. M. 2000, ApJ, 528, L37

Shinnaga, H., Moran, J. M., Young, K. H., \& Ho, P. T. P. 2004, ApJ, 616, L47

Snyder, L. E., \& Buhl, D. 1974, ApJ, 189, L31
Surcis, G., Vlemmings, W. H. T., Torres, R. M., van Langevelde, H. J., \& Hutawarakorn Kramer, B. 2011, A\&A, 533, A47

van Kempen, T. A., Wilner, D., \& Gurwell, M. 2009, ApJ, 706, L22

Vlemmings, W. 2002, Ph.D. Thesis, Leiden University

Vlemmings, W. H. T., \& van Langevelde, H. J. 2005, A\&A, 434, 1021

Vlemmings, W. H. T., van Langevelde, H. J., \& Diamond, P. J. 2005, A\&A, 434 1029

Vlemmings, W. H. T., Diamond, P. J., \& Imai, H. 2006a, Nature, 440, 58

Vlemmings, W. H. T., Diamond, P. J., van Langevelde, H. J., \& Torrelles, J. M. 2006b, A\&A, 448, 597

Vlemmings, W. H. T., Humphreys, E. M. L., \& Franco-Hernández, R. 2011, ApJ, 728,149

Waters, J. W., Kakar, R. K., Kuiper, T. B. H., et al. 1980, ApJ, 235, 57

Watson, W. D. 2002, in Cosmic Masers: From Proto-Stars to Black Holes, eds. V. Migenes, \& M. J. Reid, IAU Symp., 206, 464

Watson, W. 2008, in Cosmic Agitator: Magnetic Fields in the Galaxy

Western, L. R., \& Watson, W. D. 1984, ApJ, 285, 158

Wiebe, D. S., \& Watson, W. D. 1998, ApJ, 503, L71

Yates, J. A., \& Cohen, R. J. 1996, MNRAS, 278, 655

Zapata, L. A., Menten, K., Reid, M., \& Beuther, H. 2009, ApJ, 691, 332 